\title{
La transformation des registres de légitimation politique en Guyane
}

Une approche comparative des campagnes municipales à Cayenne et Saint-Laurent du Maroni

The transformation of the registers of political legitimation in French Guiana: a comparative approach of the municipal campaigns in cayenne and Saint-

Laurent du Maroni

Stéphanie Guyon et Marie-Emmanuelle Pommerolle

\section{(2) OpenEdition}

\section{Journals}

Édition électronique

URL : http://journals.openedition.org/plc/816

DOI : $10.4000 /$ plc. 816

ISSN : 2117-5209

Éditeur

L'Harmattan

Édition imprimée

Date de publication : 1 janvier 2010

Pagination : 37-66

ISBN : 978-2-296-1141-3

ISSN : 1279-8657

Référence électronique

Stéphanie Guyon et Marie-Emmanuelle Pommerolle, « La transformation des registres de légitimation politique en Guyane », Pouvoirs dans la Caraïbe [En ligne], 16 | 2010, mis en ligne le 06 mai 2011, consulté le 19 avril 2019. URL : http://journals.openedition.org/plc/816 ; DOI : 10.4000/plc.816 


\title{
LA TRANSFORMATION DES REGISTRES DE LEGITIMATION POLITIQUE EN GUYANE :
}

\author{
UNE APPROCHE COMPARATIVE DES CAMPAGNES MUNICIPALES A \\ CAYENNE ET SAINT-LAURENT DU MARONI
}

\author{
Stéphanie GUYON \\ Membre du CRPS \\ Université Paris I
}

\author{
Marie-Emmanuelle POMMEROLLE \\ Membre du CRPLC \\ Université des Antilles et de la Guyane
}

Villes à très forte croissance démographique ${ }^{1}$, villes interfaces entre l'espace politique national et des espaces locaux postcoloniaux, Cayenne et Saint-Laurent du Maroni, la préfecture et la sous-préfecture de Guyane, sont des sites particulièrement riches d'observation des transformations des formes de légitimation des élus locaux. Particulièrement disputées du fait de la longévité des maires en place (ou de leur parti) ${ }^{2}$ et de certains signes avant-coureurs de l'effritement de leur popularité ${ }^{3}$, les élections municipales

\footnotetext{
${ }^{1}$ Cayenne compte, selon le recensement INSEE de 1999, 54600 habitants et connaît une évolution annuelle de 2,3\% depuis 1990. Selon la même enquête, Saint-Laurent du Maroni compte une population de 19200 habitants et son évolution annuelle depuis 1990 est de 3,9\%. ${ }^{2}$ Depuis 25 ans, St Laurent est dirigé par Léon Bertrand, RPR puis UMP, qui l'a reprise au Parti socialiste guyanais (PSG) en 1983. Profane en 1982 lorsqu'il devient conseiller général, Léon Bertrand devient maire un an après et se professionnalise dans les années 1980, devenant conseiller régional en 1983, député en 1988 et enfin, consécration ultime, secrétaire d'Etat puis ministre délégué au tourisme dans les gouvernements Raffarin I, II et De Villepin. Cayenne était dirigée depuis 1965 par le PSG, parti qui détient aujourd'hui (depuis 1992) le Conseil régional, et qui a longtemps dirigé le Conseil général. La surprise de cette élection municipale 2008 réside dans la défaite, dès le premier tour, de la liste «Ensemble, franchissons un nouveau cap » dirigée par le maire sortant Jean-Claude Lafontaine, soutenu par le PSG $(36,04 \%)$, et dans la victoire de la liste «Ensemble pour changer Cayenne », dirigée par l'ancien premier adjoint du maire, Rodolphe Alexandre, exclu du PSG en janvier $2008(50,88 \%)$. Deux autres listes se présentaient: celle dirigée par Jean-Marie Taubira, «Cayenne, réalisons l'harmonie » du mouvement Walwari (7,95\%), et celle dirigée par Alain Chaumet, "Avec vous faire revivre Cayenne », se revendiquant de l'UMP mais n'ayant pas obtenu l'investiture officielle $(5,13 \%)$

${ }^{3}$ En 2007, Léon Bertrand, absent de sa circonscription depuis 2002, et même s'il réalise un score important $(59 \%)$ dans la commune dont il est resté maire, perd les élections législatives au profit de la première vice-présidente de Région apparentée PSG. Les élections municipales de 2008 interviennent donc dans un contexte différent des scrutins de 1995 - liste unique - et de 2001 - une seule liste soutenue par le PS opposée à celle du maire - qui furent très peu concurrentielles. En 2008, six listes s'affrontent (en plus de celle de Léon Bertrand,
} 
de 2008 ont permis d'observer le recours, par les listes en concurrence, à des attributs de la modernité politique empruntés à l'espace politique central, remettant en cause des pratiques anciennes et permettant de s'ajuster à des contraintes locales nouvelles. Ce fut le cas notamment de la disqualification des partis politiques (les listes sans étiquette se multipliant), de la mise en avant d'une professionnalisation politique (à travers les outils de campagne ou le recours aux compétences gestionnaires locales), ou encore de la construction d'un nouveau rapport à la population insistant sur la représentation de la «diversité » et des «communautés » composant les populations de Cayenne et de Saint-Laurent du Maroni ${ }^{4}$. C'est sur ce nouveau registre de légitimation, relativement inédit dans un espace politique longtemps dominé par un même groupe, les $\mathrm{Créoles}^{5}$, dont la particularité a d'ailleurs été d'intégrer les «nouveaux arrivants » (migrants, notamment), que nous voudrions nous arrêter, car il a été utilisé par les deux listes victorieuses, celle de Rodolphe Alexandre à Cayenne, et celle de Léon Bertrand à Saint-Laurent du Maroni. Notre propos sera de comprendre les logiques d'émergence de ce nouveau registre de légitimation.

Si le soubassement démographique de ce nouveau rapport à la population est facilement décelable (migrations massives et croissance naturelle forte des groupes non créoles), ce sont les logiques de la traduction

on trouve les listes dirigées respectivement par Serge Aimé St Aude: 15,05\% - Alain Panelle : 8,66\% - Jean-Elie Panelle : 8,95\% - Paul Persdam : 3,60\% - Dominique Larique : $1,30 \%$ ) ; cela n'empêche néanmoins pas Léon Bertrand de l'emporter dès le premier tour $(62,44 \%)$. A Cayenne, l'ancrage du PSG avait commencé à vaciller en 1995. Maire depuis cette date, Jean-Claude Lafontaine s'était vu concurrencer lors de ces élections par une liste dirigée par Christiane Taubira, députée de la première circonscription de Guyane depuis 1993, qui renouvellera sa tentative en 2001, sans succès, mais qui aura obligé dès 1995 le PSG à un second tour et imposera la présence de conseillers municipaux d'opposition.

${ }^{4}$ Nous reprenons ici les éléments de la typologie établie par Rémi Lefèbvre dans « La difficile notabilisation de Martine Aubry. Entre prescription de rôle et contraintes d'identité », Politix, $\mathrm{n}^{\circ} 65,2004,119-145$.

5 Le terme «Créole» désigne les populations issues de la colonisation qu'elles soient descendantes des colons européens ou des esclaves noirs. En Guyane, en l'absence de population créole blanche, on appelle «créole» les descendants des esclaves noirs à l'exception des populations noirs-marrons. Les Noirs-marrons ou Bushinengué sont les descendants des esclaves qui ont fui les plantations de l'ancienne Guyane hollandaise au $\mathrm{XVII}^{\mathrm{e}}$ et au XVIII ${ }^{\mathrm{e}}$ siècle et se sont réfugiés en forêt où ils ont constitué un mode de vie syncrétique. Certains groupes noirs-marrons ont une présence ancienne sur le territoire guyanais, principalement sur le fleuve Maroni. 
politique de ces mutations qui sont particulièrement intéressantes à observer, et les conditions de possibilité d'un tel changement. Nous verrons d'abord combien ce registre importé de la diversité et de la communauté peut servir à exacerber les clivages dans un contexte de fortes oppositions entre les candidats et alors même que les pratiques de campagne peuvent se ressembler. Si ce rapport aux identités politiques renouvelle le discours politique guyanais, nous verrons ensuite que seule la permanence de pratiques anciennes, même revues à la marge, peut expliquer que ce registre symbolique soit aussi efficace. Ainsi, si compétence a rimé, lors de la campagne, avec communauté, c'est que la première permet une sélectivité sociale forte des élites politiques derrière un discours toujours aussi moderne. De plus, si les «communautés » (souvent en fait, les minorités), sont mises à l'honneur, ce sont avant tout les pratiques clientélaires, certes renouvelées, qui permettent de maintenir une loyauté sans faille et de garantir la longévité politique de certaines élites politiques.

\section{Communautés, diversité, proximité : la construction controversée de nouveaux rapports à la population}

Avec la départementalisation de la Guyane en 1946, ce sont les Créoles qui composent l'élite politique guyanaise, cayennaise et saintlaurentaise. En effet, dès le XIX ${ }^{\mathrm{e}}$ siècle, les esclaves affranchis et leurs descendants qui forment la population créole s'engagent dans un processus d'assimilation à la société métropolitaine, en particulier par l'accès à la scolarisation. L'idéologie de l'assimilation hiérarchise la société guyanaise, instaurant la domination sociale et politique du groupe créole - par ailleurs longtemps démographiquement dominant - sur les Amérindiens et les Noirsmarrons dont le mode de vie aborigène est considéré comme «primitif » par l'administration coloniale et les Créoles ${ }^{6}$. Depuis les années 1970, et les nouvelles vagues de migration (d'Haïti, du Brésil, du Surinam, du Guyana), la société guyanaise se créolise moins et se compose désormais de groupes séparés et désignés par leur origine (on parle des Brésiliens, des Chinois, etc... sans savoir s'il s'agit de Français ou non). Dans l'Ouest, les migrations du Surinam et du Guyana ainsi que la forte natalité des groupes amérindiens

${ }^{6}$ Marie-José Jolivet, La question créole, Essai de sociologie sur la Guyane française, Paris, Éditions de l'O.R.S.T.OM , 1982, 503 p. 
et noirs-marrons ont accru la présence des groupes non créoles. Ces mutations démographiques importantes ont trouvé, pour la première fois de manière aussi marquée, une traduction politique lors de ces élections municipales. A Cayenne, où ces transformations de la population (et donc de l'électorat) sont le résultat de l'immigration et où la domination politique créole est forte, l'unanimité ne s'est pas faite autour de ce nouveau registre de la représentation des «communautés», dénoncée comme antirépublicaine et dangereuse pour la société. A l'encontre de ces arguments déployés par des listes plutôt menacées, ce nouveau registre communautaire a été conçu comme un «coup », et l'importation d'une modernité politique empruntée au champ politique central. Jouant de nouvelles contraintes sociales, et se réappropriant un registre moderne, ce sont les listes victorieuses qui n'ont pas hésité à construire ce nouveau rapport à la population guyanaise. Il a pu s'accompagner d'un recours à la technique de la « démocratie participative », autre outil de légitimation moderne emprunté au champ central.

\section{A - La représentation des « communautés ${ }^{7}$}

1) Des pratiques de composition des listes...

Qu'elle soit revendiquée ou dénoncée, la prise en compte de l'appartenance communautaire est un critère effectif de composition des listes pour la majorité des faiseurs de listes ${ }^{8}$, à Saint-Laurent du Maroni comme à Cayenne et quel que soit le type de liste, partisane, sans étiquette, sortante ou pas. Cette logique n'est d'ailleurs pas nouvelle, en particulier à

\footnotetext{
${ }^{7}$ Nous reprenons ici le terme communément employé en Guyane pour désigner, par opposition au groupe créole dominant, les groupes minorisés qu'ils soient issus de l'histoire coloniale guyanaise - Amérindiens, Noirs-marrons - ou de l'immigration récente Brésiliens, Haïtiens, Hmongh...

${ }^{8}$ Il n'est pas évident de reconstituer ces logiques de composition des listes puisque le discours sur la composition de la liste est un argument de campagne, néanmoins les entretiens avec les faiseurs de liste, tête de liste ou directeurs de campagne, permettent en partie d'éclairer les pratiques effectives de composition, qu'elles soient en cohérence ou non avec les discours de campagne.
} 
Saint-Laurent du Maroni ${ }^{9}$. Seules les listes Taubira et Chaumet, relativement dépourvues de ressources politiques, récusent en tenir compte. A ces exceptions près, les faiseurs de listes parlent tous de la représentation des communautés comme un obligé de la composition de la liste ; le directeur de campagne de Léon Bertrand dit ainsi :

« Donc cette liste a été faite avec beaucoup d'attention en fonction déjà de la parité puisqu'elle est obligatoire, c'est la loi, deuxièmement indépendamment de cette parité, St Laurent, c'est quand même beaucoup d'ethnies qui résident. Donc on l'a vu à l'usage, il faut un équilibre, il faut un équilibre entre toutes ces ethnies, noirs-marrons, amérindiennes, créoles pour ne citer que les plus grosses. $»^{10}$

Cette logique est ici renvoyée à la spécificité démographique de Saint-Laurent du Maroni mais elle fonctionne de la même manière à Cayenne, même lorsque les têtes de listes s'en défendent. Ainsi la liste PSG ne revendique pas de représentation communautaire, voire la dénonce. Pourtant, un organisateur de la campagne de Jean-Claude Lafontaine dit avoir consulté les listes électorales et compté le nombre d'électeurs nés au Brésil, en Chine ou en Haïti, et sélectionné de ce fait des candidats de chacun de ces groupes d'originaires. Figurent donc sur la liste PSG, une femme d'origine chinoise et une autre d'origine brésilienne. On retrouve d'ailleurs ici une constante de l'application de la parité : c'est surtout aux femmes profanes qu'incombe l'obligation de représenter les minorités. On observe également à Saint-Laurent du Maroni que sur 7 colistiers provenant des groupes amérindiens, noirs-marrons et chinois, 5 sont des femmes profanes et deux sont des hommes non profanes.

Certaines listes sans ressource politique et faiblement attractives éprouvent des difficultés à garantir cette représentation des communautés qu'elles considèrent pourtant stratégique. Ces listes ont en effet du mal à attirer des candidats en dehors du milieu d'interconnaissance de leurs leaders. Ainsi les deux listes de gauche de Saint-Laurent du Maroni, la liste

\footnotetext{
${ }^{9}$ En 1983, sur la liste de Léon Bertrand, figuraient déjà deux Amérindiens et un Noir-marron. Mais le quota implicite de conseillers municipaux et d'adjoints issus des groupes minorisés s'est élevé, il est désormais d'au moins deux conseillers et un adjoint par groupe.

${ }^{10}$ Entretien avec le directeur de campagne de L. Bertrand, Saint-Laurent du Maroni, 15 mars 2008.
} 
EUC $^{11}$ (Alain Panelle) et la liste PS (Jean-Elie Panelle) comptent quelques foyers de recrutement privilégié: les enseignants métropolitains et les Amérindiens du village Balaté ${ }^{12}$ pour la première, la section locale du PSG dont nombre d'employés du Conseil général et de l'hôpital - et du PS principalement des enseignants métropolitains - pour la seconde. Ces deux dernières listes peinent à attirer des candidats noirs-marrons qui représentent pourtant $50 \%$ de la population de la commune. La contrainte de représentation de cette communauté les oblige à faire figurer sur leur liste des personnes disposant de faibles ressources sociales et politiques (sans emploi, sans mandat associatif...).

D'autres listes faiblement attractives, comme les listes Chaumet et Taubira (Walwari) à Cayenne, disent refuser d'avoir recours à ce type de composition communautaire :

«On est contre le communautarisme, Walwari (en tout cas, dans le courant auquel $\mathrm{j}$ 'appartiens dans le mouvement), on ne fera pas venir quelqu'un parce qu'il est d'origine ci ou ça, s'il se présente sur les listes, c'est parce qu'il a la nationalité française ; et on le dit franchement, on le dit aux communautés, on est contre la partition du pays en communautés, on est pour que les gens se côtoient, (...) pour autant, on ne demande pas aux gens d'oublier leurs origines, c'est pas du tout ça. "Réalisons l'harmonie', c'est pour ça qu'on a dit ça. Par exemple, Hong Wei, il est là parce qu'il partage nos idées, pas parce qu'il est Chinois. ${ }^{13}$. Il est cependant difficile de savoir si cela ne relève pas d'une rationalisation a posteriori d'une impossibilité pratique d'attirer des membres de différentes communautés. Le mouvement Walwari, s'il a toujours tenu un discours fermement anti-communautariste, a eu recours lors d'autres scrutins aux pratiques dénoncées ici. Ainsi aux régionales de 2004, une Amérindienne et un Amérindien figuraient respectivement en $5^{\mathrm{e}}$ et $6^{\mathrm{e}}$ positions sur la liste Walwari. Tous deux non encartés à Walwari, ils n'avaient quasiment pas fait campagne et ne figuraient plus sur la liste après la fusion entre les deux tours avec une autre liste.

\footnotetext{
${ }^{11}$ Ensemble Unis pour le Changement.

${ }^{12}$ La cheffe coutumière du village Balaté est la candidate EUC aux élections cantonales tandis que son mari, enseignant, est $5^{\mathrm{e}}$ sur la liste aux élections municipales.

${ }^{13}$ Entretien avec Jean-Marie Taubira, tête de liste Walwari, 21 mars 2008.
} 
La représentation des communautés apparaît donc avec d'autres critères comme un obligé de la composition des listes à Cayenne et SaintLaurent du Maroni. Néanmoins toutes les listes n'en font pas le même usage. Pour certaines listes qui se sont approprié le thème de la diversité, elle constitue l'un des principaux registres de légitimation. D'autres listes au contraire la dénient et en usent pour disqualifier leurs adversaires.

\section{2) ... aux discours de campagne}

A Cayenne, un clivage net s'est construit durant la campagne autour de la représentation communautaire. Revendiquée par la liste de Rodolphe Alexandre, elle est rejetée, au nom de principes idéologiques, par les trois autres listes. Ce clivage permet bien sûr aux listes menacées de s'appuyer sur des «valeurs » qu'elles lient à leur engagement partisan à gauche (pour les listes PSG et Walwari). Le débat a donc été intense, dans les meetings et sur les plateaux télévisés autour de cette question. A Saint-Laurent du Maroni en revanche, la représentation communautaire fait consensus, mais Léon Bertrand, maire sortant UMP qui dispose des réseaux les plus étendus dans les différentes communautés, est celui qui en use le plus.

- une représentation explicite des communautés à travers le nouveau langage de la diversité

Dans la liste de Rodolphe Alexandre à Cayenne et celle de Léon Bertrand à Saint-Laurent du Maroni, la représentation communautaire est explicite tant dans la présentation de la liste que pendant la campagne, lors des porte-à-porte et réunions publiques... Dans l'un des tracts de présentation de la liste, Rodolphe Alexandre développe l'idée qu'il se fait de Cayenne : «Une ville multiculturelle dans laquelle les communautés vivent en fraternité et en solidarité ». De la même manière, Léon Bertrand, lors d'une allocution sur la place du marché de Saint-Laurent du Maroni, affirme : "J'ai toujours considéré que Saint-Laurent du Maroni était une ville tout à fait particulière, une mosä̈que culturelle pluriethnique qui mérite que toutes les communautés soient valorisées, qu'elles existent, qui mérite qu'elles soient respectées, reconnues, c'est pourquoi notre liste est très 
représentative.» ${ }^{14}$. Pour la première fois, la représentativité « communautaire » de la liste, la «diversité » sont mises en avant en centreville, auprès de l'électorat créole et pas seulement dans les villages et quartiers amérindiens et noirs-marrons de St Laurent du Maroni. Lors du débat télévisé sur Télé Guyane (4 mars 2008) entre les quatre têtes de liste cayennaises, Rodolphe Alexandre n'hésite pas à avancer que «sa liste représente toutes les communautés» et qu'elle est «la seule liste où se trouvent des personnes d'origine brésilienne, haïtienne, métropolitaine, guadeloupéenne, martiniquaise ».

A Cayenne, la liste de Rodolphe Alexandre a délibérément choisi de mettre en avant la représentation des diverses « communautés », qui sont en fait les personnes issues de l'immigration. Son équipe a ainsi fait le décompte des électeurs nés à l'étranger et leur a envoyé un courrier ciblé. L'un des chargés de la logistique explique: "On a extrait [de la liste électorale] la liste des Français nés à l'étranger, Haïti, Brésil, etc... On a trouvé 795 Haïtiens, 395 Brésiliens. On leur a envoyé des courriers personnels $^{15} »$. Cette représentation explicite est l'un des arguments de campagne lors des porte-à-porte. Ainsi, à l'occasion d'une tournée, trois colistiers distribuent un tract à un couple d'Haïtiens, assis devant sa baraque en tôle. Alors que le couple est sceptique lorsque le mot «changement » est prononcé, les colistiers insistent sur le fait qu'ils auront désormais deux représentants à la Mairie et qu'ils devront leur faire passer des messages ${ }^{16}$. La fonction unique de «représentant communautaire » de ces colistiers est patente : lors de cet échange, les trois colistiers créoles sont capables de ne citer nommément qu'un seul de leurs colistiers d'origine haïtienne, ne connaissant pas le nom du second. A Saint-Laurent du Maroni, l'interconnaissance est plus forte entre les candidats, mais là aussi les colistiers noirs-marrons et amérindiens ont principalement pour mission de représenter leur communauté. Ils sont présents dans toutes les réunions et porte-à-porte dans les quartiers amérindiens et noirs-marrons. Ils s'y présentent comme les relais de leur communauté au sein de la municipalité :

« Je suis adjoint et c'est grâce aux adjoints, que Biatan [nom de Léon Bertrand en langue noir-marron] peut faire des choses

\footnotetext{
${ }^{14}$ Léon Bertrand, place du marché, Saint-Laurent du Maroni, 8 mars 2008.

${ }^{15}$ Discussions lors du meeting du $1^{\mathrm{er}}$ mars 2008, Cayenne.

${ }^{16}$ Observations, Cayenne, 6 mars 2008.
} 
pour les gens du fleuve [Amérindiens et Noirs-marrons]; c'est en consultant ses adjoints qu'il va mettre en place son programme... $»^{17}$

Après l'élection de la liste Rodolphe Alexandre à Cayenne et celle de Léon Bertrand à Saint-Laurent du Maroni, certaines délégations sont données en fonction du rapport des conseillers municipaux à une communauté : à Cayenne, un élu d'origine chinoise est désormais $6^{\mathrm{e}}$ adjoint, en charge du commerce et donc des rapports avec les commerçants chinois ; à Saint-Laurent du Maroni, une élue amérindienne, est $7^{\mathrm{e}}$ adjointe en charge de l'aménagement de villages (amérindiens) et des relations avec les autorités coutumières.

Rodolphe Alexandre et Léon Bertrand font tous deux usages du champ lexical de la diversité, importé du champ central. Léon Bertrand, dont l'insertion dans le champ central est forte - ancien député, ancien ministre utilise ainsi pour la première fois lors de ce scrutin le terme de "diversité », qui figure dans la dénomination même de sa liste, «Bâtir Saint Laurent dans la diversité ». On observe ainsi le déplacement sémantique par rapport à la liste qu'il conduisait aux régionales de 2004 «Redonnons des couleurs à la Guyane ». Nous faisons l'hypothèse que cet emprunt au champ central permet de légitimer auprès des Créoles la représentation des groupes minorisés de Saint-Laurent du Maroni, utilisant un terme qui l'euphémise et l'inscrit dans un processus plus général puisqu'il concerne également le territoire métropolitain. La mise en avant de la représentation des communautés s'effectue néanmoins dans des contextes différents à Cayenne et Saint-Laurent du Maroni. Rodolphe Alexandre à Cayenne est un outsider, premier adjoint sortant, sans investiture partisane, il joue un coup en axant ainsi sa campagne sur un thème importé de métropole qui le distingue du maire sortant. Les colistiers de Rodolphe Alexandre interrogés sur l'accent mis sur ces « communautés » répondent avant tout en termes stratégiques, et ne récusent pas le terme de communautarisme. Ils ont une vision très pragmatique de cette représentation communautaire. Léon Bertrand, maire depuis 1983, investi par l'UMP dont il est le chef de file en Guyane, dispose de nombreuses ressources, partisanes et municipales et la mise en scène de la représentation communautaire permet avant tout de faire la démonstration de

\footnotetext{
${ }^{17}$ Colistier UMP, adjoint sortant. Réunion au village Sparouine, 7 mars 2008.
} 
ses ressources locales, de son ancrage dans le territoire communal et en particulier au sein des différentes communautés.

- le déni de la représentation communautaire

Ce registre de légitimation est beaucoup plus délicat à Cayenne, pour au moins deux raisons : les rapports de force politiques y sont relativement indéterminés en début de campagne - les coups politiques y sont donc déterminants - et surtout, la représentation des communautés équivaut à Cayenne à la représentation des personnes issues de l'immigration, sujet parmi les plus sensibles politiquement dans ce département d'outre-mer. Mais au-delà des discours qui stigmatisent le «communautarisme », les pratiques de campagne prennent en compte la nouvelle démographie cayennaise.

Lors des réunions publiques, et lors des entretiens, le discours de la liste conduite par Jean-Claude Lafontaine est violemment anticommunautaire et insiste, c'est l'un de ses slogans, sur "l'unité dans la diversité », diversité qu'il entend surtout en termes géographique et social comme nous le verrons. Néanmoins, le maire sortant se rend dans des associations d'originaires, déplacements au cours desquels il récuse le communautarisme tout en utilisant des arguments ciblés vers son public. Ainsi, dans une maison particulière où sont réunies une quarantaine de personnes d'origine haïtienne, Jean-Claude Lafontaine fustige le communautarisme tout en rappelant sa propre origine «extérieure »: «Mon grand père est né en Martinique. Mais moi je suis Guyanais. On ne doit pas se développer séparément. Dans la bataille électorale on divise, avant c'était les gens de la ville et les gens de la campagne. Mais on est tous de Cayenne ${ }^{18}$. Il utilise également des arguments adaptés à son audience: lors de cette même réunion avec des Haïtiens et originaires d'Haïti en effet, il stigmatise le racisme des Créoles («Certains Créoles disent qu'il y a trop d'Haïtiens, mais tout homme est un homme »); il rappelle, avec un sourire entendu, le vote Sarkozy de son adversaire, Rodolphe Alexandre, et l'accuse d'avoir des positions fluctuantes sur l'immigration haïtienne («Rodolphe Alexandre, il a deux discours : un en créole haïtien, et un en créole guyanais

${ }^{18}$ Observations, Cayenne, 6 mars 2008, retranscrit en français à partir d'un mélange de français et de créole guyanais. 
où il dit qu'ils doivent partir »). Interpellé sur la question de l'obtention extrêmement difficile de papiers, il promet d'aller voir le préfet.

Cette forme de campagne ciblée ne correspond donc pas au discours idéologique tenu dans les grands meetings ou dans les médias. D'ailleurs, n'assumant pas de présenter des candidats communautaires lors de la présentation de sa liste, le maire sortant fait le lien entre la représentation des communautés et la représentation des quartiers. Ce sont avant tout des représentants de quartiers qu'il met en avant, combinant ainsi ces deux impératifs. Il dit ainsi de la jeune femme d'origine brésilienne de sa liste qu'elle habite le «village brésilien» et que «Ca, pour moi, c'est important $»^{19}$.

\section{B - La représentation territoriale et sociale}

Selon la composition spatiale de la ville en effet, représentation des quartiers et représentation des communautés peuvent se recouper plus ou moins. Ainsi, à Saint-Laurent du Maroni, étant donnée la forte ségrégation spatiale, connaissance des quartiers rime nécessairement avec connaissance des communautés. Néanmoins, dans les deux villes, au-delà des caractéristiques identitaires des «communautés », la représentation sociale et territoriale continue d'être un registre majeur de légitimation des candidats sortants. A Cayenne, c'est la liste de Jean-Claude Lafontaine qui reflète cette attention portée aux quartiers populaires, héritage d'un parti ancré dans ces quartiers et de la trajectoire de la tête de liste. Sa manière de parler de «diversité sociale et géographique » est aussi un moyen de marquer le clivage avec son adversaire, ancien adjoint au maire. Rapport de forces politique et registres de légitimation se construisent de concert. A SaintLaurent du Maroni et à Cayenne, les deux maires sortants insistent également, et de manière classique, sur la proximité qu'ils entretiennent, de par leur mandat, avec leurs habitants, et accusent les listes adverses de présenter des candidats alibis, censés incarner cette proximité sociale avec les populations. Afin de contourner ce déficit de «proximité », la technique de la démocratie participative est évoquée par le challenger cayennais. Sorte de «proximité professionnalisée », elle est le signe d'une attention aux

${ }^{19}$ Observations, Cayenne, 20 février 2008. 
populations et d'une modernité politique importée et vient compenser la faiblesse de l'expérience de proximité.

\section{1) L'incarnation des quartiers populaires}

Lors de la présentation de la liste de Jean-Claude Lafontaine, l'accent est mis sur la représentation des «quartiers » (Sud) de Cayenne, c'est-à-dire des quartiers populaires. Placés en deuxième moitié de liste, les représentants de ces quartiers (cinq environ) combinent aussi une autre caractéristique importante, celle de la jeunesse. Typiquement, la $22^{\mathrm{e}}$ sur la liste est présentée comme une jeune femme, assistante sociale, dont la famille est active dans le quartier Bonhomme. Lors d'une réunion chez les parents de la colistière, où sont réunis une quarantaine de jeunes (15-20 ans), le maire sortant explique pourquoi il l'a choisie: "c'est pour montrer que c'est pas parce qu'on est des quartiers périphériques qu'on est des citoyens de seconde zone. Elle représente une partie de la population. Elle représente la jeunesse et la réussite. $»^{20}$

De la composition de la liste aux tournées dans les quartiers, le maire sortant a choisi un discours tourné vers les quartiers populaires auxquels il veut redonner leur dignité («Je veux donner aux habitants des quartiers le bien être, leur donner la fierté d'habiter Cayenne $\left.»^{21}\right)$. Lors de la soirée de présentation de la liste $\mathrm{PSG}^{22}$, son directeur de campagne met en avant le «caractère populaire, ancré dans la réalité guyanaise» de la liste, et le secrétaire général du PSG insiste : "Il faut se différencier de ceux qui font du porte-à-porte dans les quartiers et qui ensuite retrouvent leurs villas bien climatisées, loin des inondations ». Lors d'une réunion avec des Haïtiens et personnes d'origine haïtienne à Baduel, le maire sortant assène: "Eux [la liste de R. Alexandre], ils ont des patrons qui exploitent les gens». Effectivement la liste PSG serait plus «populaire» que la liste Rodolphe Alexandre, selon l'un(e) de ses colistier(e)s bien placé(e)s, qui regrette d'ailleurs qu'il n'y ait pas eu davantage de personnalités représentant les cantons du centre-ville " petit bourgeois ». Les résultats de la liste y sont d'ailleurs plus mauvais que dans le reste de la ville : $26 \%$ et $28,6 \%$ dans les

${ }^{20}$ Observations, Cayenne, 6 mars 2008.

${ }^{21}$ Télé Guyane, « Paroles de campagne », 28 février 2008.

${ }^{22}$ Observations, Cayenne, 20 février 2008. 
deux bureaux de vote du centre ville. Lors de la campagne, le maire sortant dénonce les orientations de son adversaire qui souhaite embellir ce centreville: "Alexandre dit: 'avant 2008, le boulevard Jubelin [boulevard du centre-ville] ce sera les Champs Elysées'. Mais nous, on ne va pas mettre le boulevard Jubelin dans nos priorités. Je ne veux pas de la disparité entre les quartiers $»$. Durant toute sa dernière mandature, il a en effet privilégié l'action dans les quartiers populaires, au détriment du centre-ville. Cette orientation semble pouvoir se rapporter à la trajectoire de la tête de liste, ancien médecin généraliste installé dans un quartier Sud (quartier dans lequel il arrive en tête avec $46 \%$ des voix). Qualifié de personne «bien à gauche » par l'une de ses anciennes adjointes, il est entré en politique dans les années 1970, et conserve un discours marqué à gauche que l'on retrouve rarement aussi nettement dans les discours de ses colistiers ou du PSG. ${ }^{23}$.

Cette incarnation sociale de la population s'oppose à l'accent mis sur les communautés par l'adversaire principal de Jean-Claude Lafontaine dans le cas de la ville de Cayenne. En fait, c'est l'opposition entre représentation sociale et représentation identitaire qui se joue ici, et qui permet de marquer et de renforcer le clivage entre ces deux anciens alliés politiques, dont l'un a été exclu du PSG, parti de gauche et se revendiquant désormais «sans étiquette».

\section{2) La proximité : entre intimité et technique politique}

Un autre moyen de renforcer les clivages et de favoriser les élus sortants, est de jouer sur la proximité de ces derniers avec les populations et la connaissance intime du territoire qu'ils détiendraient du fait de leur expérience. La proximité serait une arme redoutable aux mains des sortants, qui se voient néanmoins concurrencés désormais par ceux qui les défient et qui ont intégré le nouveau vocabulaire politique de la «démocratie participative ».

${ }^{23}$ «Ma conception politique, c'est à gauche, ce sont les socialistes. C'est défendre ceux qui souffrent le plus. R. Alexandre, sur la liste, il a un avocat qui gagne, mais nous sur notre liste, ce sont des mouns [personnes en créole haïtien] qui vivent dans les quartiers. Nous, on a une conscience de classe, de la classe défavorisée », Discours du maire sortant devant une assemblée de personnes haïtiennes ou d'origine haïtienne à Cayenne. Notes de terrain, 6 mars 2008. 
Les deux maires sortants n'ont eu de cesse de rappeler leur connaissance intime des quartiers et de leurs populations. Dans un tract, Jean-Claude Lafontaine rappelle :

«Cette transformation [des quartiers], nous l'avons engagée ensemble dans le cadre de la concertation avec les comités de quartier qui ont largement contribué à l'élaboration de ce projet municipal (...) Je n'ai pas attendu cette campagne électorale pour aller à votre rencontre, pour débattre de vos idées, écouter vos préoccupations. »

Léon Bertrand utilise des arguments similaires: «Vous me connaissez, vous savez que je vous ai toujours reçus, qu'il est facile de venir me parler». Il répond aux attaques de ses adversaires qui l'accusent de vouloir continuer à vivre à Paris :

«C'est un mensonge que je ne serai pas là parce que je suis nommé inspecteur général de l'éducation nationale, je serai là et je vais même augmenter mon nombre de permanences, avant je recevais le mardi et le vendredi, désormais, je recevrai les mardi, mercredi et vendredi ».

A l'attention passée et future portée aux populations, les deux maires sortants ajoutent une connaissance pointue des quartiers de la ville qu'ils souhaitent continuer à diriger. Lors de ses tournées dans les quartiers, JeanClaude Lafontaine cherche régulièrement à parler avec précision des installations réalisées (terrain de sport) et de celles qu'il reste à améliorer (nouvelle voirie). Il n'hésite pas non plus à montrer qu'il est à l'écoute des quartiers puisqu'il a recruté sur sa liste un certain nombre de jeunes qui en sont issus et qu'il aurait rencontrés «lors de ses parcours professionnels et sociaux ». C'est ainsi qu'il présente publiquement Létard Oswald, $30^{\mathrm{e}}$ sur sa liste : "Il m'en a fait voir dans son quartier, à Eau Lisette. C'est l'exemple de ce que devrait être la vie politique, faire des réunions de quartier dans lesquelles les gens vous interpellent $\gg$.

A Saint-Laurent du Maroni, la proximité est synonyme de bonne connaissance des communautés du fleuve Maroni, et passe en particulier par 
la maîtrise du sranan tongo ${ }^{24}$. C'est ce que souhaite démontrer le maire sortant :

«A part Paul Persdam et moi-même, aucun des candidats aux élections municipales, je dis bien aucun, ne connaît la langue, alors s'ils venaient à être élus, comment feront-ils, comment pourra-t-il recevoir la population, comment pourrait-on avoir un maire qui ne peut pas comprendre sa population, comment ferez vous pour lui parler? $»^{25}$

Léon Bertrand se pose comme le seul candidat respectant les Noirsmarrons et Amérindiens. Au village amérindien Pierre, il affirme ainsi :

«Ils viennent avec leur musique à fond, ils ne respectent pas la tranquillité des villages, ils ne connaissent pas. Nous, nous ne venons pas avec la musique, nous ne sommes pas des sauvages, nous faisons des réunions pour parler avec les gens. Nous sommes les seuls à faire des réunions, les autres viennent mais ne font pas de réunions $»^{26}$.

Comme, on l'a vu, les colistiers issus des communautés sont ainsi un gage de proximité et de compréhension des enjeux locaux :

«S'il n'y avait pas de personnes bushinengué [noirs-marrons] avec lui, il ne pourrait pas faire cela et il ne pourrait pas aider ceux qui sont dans les villages comme Sparouine, éloigné de Saint Laurent. C'est les adjoints qui font le relais ${ }^{27}$

Les militants UMP accusent les autres listes de ne pas avoir cette connaissance et de n'avoir que des Amérindiens et des Noirs-marrons «alibi » sur leur liste.

L'intimité entre l'élu et la population ne pourrait donc pas s'inventer à travers ces alibis de proximité. Néanmoins, l'importation d'un vocabulaire moderne, celui de la «démocratie participative » est pratiquée, notamment par ceux qui ne bénéficient pas de cette intimité avec la population,

\footnotetext{
${ }^{24}$ Le sranan tongo est la langue créole du Surinam que parle la majorité des Noirs-marrons et des Amérindiens de Saint-Laurent du Maroni en plus de leur langue maternelle.

${ }^{25}$ Conférence publique dans le quartier noir-marron de la Charbonnière, Saint-Laurent du Maroni, 7 mars 2008.

${ }^{26}$ Réunion publique au village Pierre, 6 mars 2008.

${ }^{27}$ Colistier UMP, Réunion au village de Sparouine, 7 mars 2008.
} 
construite au fil des mandats. C'est ainsi que Rodolphe Alexandre ne se prive pas de mobiliser le terme de "démocratie participative», et de l'évoquer comme une technique de gestion locale efficace et se combinant parfaitement avec la compétence revendiquée de sa liste (cf. infra). Evoquée dans sa lettre aux militants PSG de la section de Cayenne, développée dans l'un de ses premiers tracts de campagne intitulé «démocratie participative » détaillant les dossiers traités en comités de quartier et annonçant la création d'un site internet interactif, la démocratie participative est aussi le sujet du tract comparatif publié par la liste de Rodolphe Alexandre sous le titre : «2 hommes -2 programmes -2 visions », dans lequel sont mis en parallèle « Jean Claude Lafontaine, pensée unique $=\mathrm{Je}$ » et «Liste conduite par R. Alexandre, Démocratie participative $=$ Nous $»$. Devenu un élément ordinaire du discours politique, ce terme permet de pallier un manque d'expérience concrète de la «proximité » tout en mettant en avant une forme de modernité politique. Conseillé par un directeur de campagne professionnel ${ }^{28}$, Léon Bertrand n'hésite d'ailleurs pas à évoquer successivement sa connaissance réelle d'une ville qu'il dirige depuis 1983 et sa volonté de mettre en place une véritable démocratie participative, insistant ainsi à la fois sur l'expérience vécue et le savoir-faire de la proximité.

\section{Les conditions de possibilité de nouveaux registres de légitimation}

Les transformations et les ajustements relatifs aux modalités de représentation des populations se sont appuyés sur la permanence relative d'autres aspects des rapports entre représentants et électeurs. Le renouvellement des listes et des discours et pratiques de campagne n'a en effet pas été total. Au contraire, la mise en avant de registres inédits a pu occulter la persistance de pratiques anciennes - modifiées à la marge. A Cayenne, l'intégration des «communautés» s'est accompagnée d'un discours sur la mobilisation de « compétences », qui a permis de justifier une sélectivité sociale forte au sein de la liste victorieuse et le maintien de

\footnotetext{
${ }^{28}$ Son directeur de campagne est son ancien attaché parlementaire qui a une longue carrière de collaborateur d'élu et de directeur de campagne. Il a été formé entre 1995 et 2002 par l'équipe de communication de Jacques Chirac à la direction de campagne. Entretien avec le directeur de campagne du maire, Saint-Laurent du Maroni, samedi 15 mars 2008.
} 
réseaux de notabilité. A Saint-Laurent du Maroni, les pratiques clientélaires maîtrisées par le maire sortant sous-tendent sa victoire. Néanmoins, comme à Cayenne, le «clientélisme » se voit aujourd'hui dénoncé publiquement, par les listes minoritaires mais aussi par les maires sortants qui nient recourir à ces pratiques - parce que le contexte matériel ne s'y prête plus (à Cayenne), ou parce qu'il porte trop la marque de la domination subie par des populations-clientèles justement revalorisées par le discours communautaire. Entre disqualification et persistance relative, l'échange clientélaire classique voit ainsi ses frontières se rétrécir et sa légitimité vaciller dans le contexte d'agglomérations en croissance et d'importation de certains attributs de la modernité politique.

\section{A - Le discours sur la compétence et la sélection sociale et politique}

A côté de la représentation des «communautés », l'autre pilier de la campagne de Rodolphe Alexandre à Cayenne a été l'accent mis sur la constitution d'un «bloc de compétences». Cette expression a été le leitmotiv de sa campagne, mettant en avant les qualités politiques et professionnelles de ses colistiers. La reconnaissance publique de leur compétence et de leur expérience s'est combinée de manière implicite, mais lisible par les électeurs, avec des réputations et des notoriétés familiales. Ainsi, compétence et notabilité allaient de paire, conduisant à un renouvellement relatif des candidats et du futur personnel politique.

La composition de la liste et le discours accompagnant celle-ci marquent, en effet, la volonté de recourir à des personnes expérimentées politiquement (ayant déjà détenu un mandat électif) et surtout des personnalités du monde économique et social, formant ce «bloc de compétences ». Ancien premier adjoint et menant une liste «d'ouverture », Rodolphe Alexandre a réuni sur sa liste huit conseillers municipaux sortants : 7 sortants de la majorité PSG (le $1^{\text {er }}$ adjoint, 3 membres du PSG et 3 élus au nom de «l'ouverture » de la liste PSG en 2001) et 1 sortant de l'opposition (liste soutenue en 2001 par Walwari). En outre, sa liste comporte, en bonne place, des colistiers détenteurs d'autres mandats électifs : on y trouve en effet trois conseillers régionaux. Surtout, ce sont les qualités professionnelles des colistiers qui sont mises en avant : on y trouve quelques membres dirigeants de la Chambre de commerce et d'industrie de 
Guyane, de la Confédération générale du patronat des petites et moyennes entreprises de Guyane et d'anciens président(e)s de la jeune chambre économique, ainsi que quelques personnalités du milieu social et associatif. A travers eux, ce sont donc les capacités de la future «équipe» à comprendre les enjeux municipaux qui sont mises en avant ${ }^{29}$.

Ce choix des colistiers permet parallèlement de mobiliser des réseaux de notabilité familiale, politique et professionnelle qui confèrent à la liste une aura certaine, et qui permettent au candidat à la mairie de s'attacher des réseaux de soutien solidement ancrés dans les milieux sociaux privilégiés de Cayenne. La liste de Rodolphe Alexandre comprend ainsi un certain nombre de membres de «grandes familles politiques » cayennaises. On peut citer les représentants de la famille Horth (M-L Phinéra-Horth dont le père a été président PSG du Conseil général et dont le frère est président de l'UMP - et Gil Horth, son cousin), ceux de la famille Prévot (Joëlle Prévot, dont l'oncle est l'ancien vice-président du Conseil général et dont le grand-père a été sénateur de Guyane, et Liliane Prévot). La notoriété familiale de la famille Prévot, et de sa représentante, Joëlle, est flagrante lors des visites de porte à porte. Lors de l'une de ses tournées dans le quartier « les chemins de Baduel » (quartier résidentiel où se nichent des bidonvilles habités principalement par des Haïtiens), Joëlle Prévot rencontre plusieurs membres de sa famille (résidant dans de grandes villas) et un tiers des personnes rencontrées la connaissent, du fait de ses liens familiaux. De l'interconnaissance au vote, le pas est vite franchi, comme le montrent ces paroles d'un homme rencontré lors du porte-à-porte : «Ah vous êtes Joëlle, je connaissais bien Jean-Pierre [son oncle récemment décédé, ancien président de la $\left.\mathrm{CCIG}^{30}\right]$ (...) Je vais le dire à ma femme, elle va voter pour vous avec les deux mains ${ }^{31}$. Certains colistiers ou soutiens publics combinent également plusieurs types de notabilité, qui ne sont pas forcément mis en avant publiquement : chef d'entreprise, le $5^{\mathrm{e}}$ colistier est président du Crédit Mutuel (10000 épargnants en Guyane) et son père est un ancien

\footnotetext{
${ }^{29}$ La question de la compétence est aussi abordée par ce candidat, et d'autres, à travers leurs capacités à se positionner dans d'autres espaces politiques et institutionnels : intercommunalité, relations privilégiées avec les milieux de décision métropolitains, etc... Faute de place, nous ne développerons pas cet aspect ici.

${ }^{30}$ Chambre de commerce et d'industrie de la Guyane

${ }^{31}$ Observations, Cayenne, 6 mars 2008.
} 
Conseiller régional du parti des $\mathrm{FDG}^{32}$. Le président du comité de soutien de la liste est un ancien président de la CCIG et fils d'un ancien maire UNR de Cayenne, à qui le PSG a ravi la mairie, en 1965. La seule notabilité professionnelle peut également être mobilisée : un colistier, jeune homme nouveau en politique, employé au Conseil général, bénéficie de la notoriété de son père médecin; deux avocats connus à Cayenne figurent en très bonne place sur la liste. Sans l'expliciter publiquement, la volonté a été de placer, au moins parmi les 10 premiers de la liste, des notables : élus, membres de famille connues ou professionnels reconnus.

Notoriété familiale et sélection sociale relativisent ainsi le renouvellement affiché par la reconnaissance des communautés. Cette nuance se trouve confortée par le choix de rassembler sur une liste pourtant citoyenne et non partisane des membres éminents (élus, dirigeants) de divers partis. On trouve donc, en plus des sortants PSG, douze membres des FDG (dont le président et le secrétaire général), et des membres de l'UMP. Alors que la tête de liste a insisté plusieurs fois sur le caractère apolitique de l'élection municipale, l'appartenance partisane de ses colistiers n'a jamais été mise en avant durant la campagne ; mais elle permet, implicitement, de structurer une liste bénéficiant des (maigres) réseaux de mobilisation partisans et d'entretenir des relations avec l'élite politique guyanaise (le sénateur de Guyane et l'ancien président du conseil général sont cités et remerciés à plusieurs reprises lors des meetings de ce candidat).

A Saint-Laurent du Maroni, la compétence de la liste du maire sortant est également présentée comme un atout mais, du fait de la composition sociale de la population, les critères de la compétence ne sont pas identiques à ceux de Cayenne. De plus, si ce registre permet de reconduire les sortants et d'intégrer des notables, il est aussi un moyen d'adapter le recrutement du personnel politique aux exigences politiques définies par le nouveau rapport à la population, et notamment aux communautés : il s'agit, au nom de la compétence (et de l'incompétence de certains) d'écarter des élus trop ouvertement hostiles aux autres groupes ou trop ouvertement clientélistes.

Si les colistiers de Léon Bertrand ne présentent pas les mêmes caractéristiques professionnelles que ceux de la liste de Rodolphe Alexandre,

${ }^{32}$ Front Démocratique Guyanais. 
d'autres formes de compétence sont mises en avant : les sortants reconduits sur la liste ${ }^{33}$ sont présentés comme les plus compétents et l'expérience professionnelle des profanes est pensée comme facilement convertible en compétence de gestion municipale ${ }^{34}$. Ce recrutement social et politique n'obéit pas aux mêmes règles que celles décelées dans la composition de l'autre liste victorieuse. En effet, à la différence de Cayenne, Saint-Laurent du Maroni qui n'était qu'un bourg jusqu'à la fin des années 1970, a longtemps été marginale sur la scène politique régionale, dominée par Cayenne, et ne compte pas de «grandes familles politiques». Depuis la défaite du directeur de l'hôpital aux municipales de 1971, les notables sont également peu présents au Conseil municipal et ont de plus déserté la ville au moment de la crise industrielle qui a affecté Saint-Laurent du Maroni dans les années 1980. Les grandes familles de notables commerçants ne comptent quasiment plus de représentants dans la ville à l'exception de quelques personnes âgées. Pour autant, les logiques familiales existent bel et bien dans la composition de la liste de Léon Bertrand dans laquelle on peut identifier des membres de familles anciennement loyales à la droite issues de la classe moyenne ascendante créole de Saint-Laurent du Maroni. En l'absence de notables issus de familles saint-laurentaise, Léon Bertrand a également choisi deux colistiers d'origine antillaise anciennement établis dans la ville qui ont acquis par leurs responsabilités dans l'éducation nationale - une principale de collège, un directeur d'un grand groupe scolaire - une certaine notabilité. La principale de collège à la retraite, occupe ainsi la deuxième position sur la liste.

$\mathrm{Si}$, marginalement, le registre de la compétence permet cette sélection sociale, ce n'est pas son objectif majeur. Il s'agit surtout d'écarter,

\footnotetext{
${ }^{33} 14$ sortants sur la liste de Léon Bertrand dont une majorité d'anciens proches du maire (6 depuis 1983, 2 depuis 1989, 2 depuis 1995 et 4 depuis 2001).

34 « $\mathrm{S}$ : les personnes nouvelles, vous les avez choisies comment?

Surtout sur un critère de compétence et également de personnes qui ont l'habitude de fonctionner dans le milieu associatif.

$\mathrm{S}$ : certains n'ont jamais eu de responsabilités politiques?

C'est comme pour les études, ce qui est important, c'est la capacité des personnes à s'adapter à une délégation. Pour prendre l'exemple de Mme Bardury, c'est quelqu'un qui a un certain âge qui a eu la gestion d'un collège, en connaît les difficultés... Elle est vraiment passée dans son parcours professionnel par toutes les phases, et c'est quelqu'un qui va avoir cette capacité très vite à pouvoir s'adapter à une délégation. Ils pourront s'approprier le sujet. » Entretien avec le directeur de campagne de Léon Bertrand, samedi 15 mars, St Laurent du Maroni.
} 
au nom de l'(in)compétence ceux qui ne pourraient justifier d'une telle qualité, des candidats dont le profil et les pratiques ne correspondent plus aux nouveaux registres de légitimation développés par la liste du maire sortant. Ainsi, il est désormais nécessaire de concilier la compétence avec un échange clientélaire rénové. C'est ainsi qu'on assiste à une rétrogradation des anciens élus clientélistes et peu compétents au profit d'élus plus diplômés.

\section{B - Disqualification ou hybridation de l'échange clientélaire : modalités d'appropriation des registres de la compétence et de la diversité.}

A Cayenne, le registre de la diversité et de la compétence, vient se substituer aux liens de clientèle qui constituaient l'un des principaux modes de rapport à la population. La diversité et la compétence mises en exergue par la liste Rodolphe Alexandre disqualifient les pratiques clientélistes de la municipalité sortante. A Saint-Laurent du Maroni, au contraire, la légitimation par la diversité vient renforcer la force des liens de clientèle puisque les rapports clientélaires constituent le mode d'insertion privilégié des communautés amérindiennes et noirs-marrons. Le modèle de la compétence ne remet pas non plus en question la centralité de l'échange clientélaire, mais il contribue à le faire évoluer, l'échange clientélaire n'étant pas contradictoire avec une certaine professionnalisation.

\section{1) Un échange clientélaire en déclin}

Le choix de la liste Jean-Claude Lafontaine d'orienter le discours de campagne vers les quartiers est le résultat d'une volonté de la tête de liste et d'une histoire politique locale : le PSG s'est maintenu à Cayenne grâce à la distribution collective et individuelle de ressources municipales. On peut faire l'hypothèse qu'on assiste au déclin de cette forme d'échange clientélaire classique : les ressources de la ville s'épuisent - même si la rumeur voulait que 60 personnes aient été embauchées juste avant les élections - et le maillage d'une ville en pleine croissance démographique semble désormais impossible. Bien sûr, on a pu trouver les résidus de cette relation clientélaire. Lors d'un porte-à-porte avec la liste de Rodolphe 
Alexandre ${ }^{35}$, les colistiers s'arrêtent devant une palissade en tôle sur laquelle est collée une affiche de la liste conduite par Jean-Claude Lafontaine. Alors que l'un des colistiers frappe, un vieux Monsieur passe la tête dans l'embrasure de la porte. Les colistiers lui demandent : «Alors, vous avez mis cette affiche, vous savez déjà pour qui vous allez voter? (...) Vous voulez notre tract?». Méfiant, l'homme répond timidement «Non, non»; ses interlocuteurs insistent et il finit par lâcher qu'il travaille pour la Mairie de Cayenne. L'enthousiasme des trois autres retombe face à cet aveu. Ils le saluent et s'en vont, sans même tenter de le convaincre.

Mais ces liens de loyauté entre agents de la Mairie et PSG, manifestation des pratiques clientélistes de la Mairie, sont constamment déniés par la tête de liste : à plusieurs reprises, dans les quartiers, le Maire dit qu'il serait malhonnête de sa part de promettre des emplois à deux jours des élections, tout en laissant des portes ouvertes à ceux qui demandent personnellement à être aidés. Lors d'une visite de quartier à Monlucas ${ }^{36}$, grande cité de Cayenne, une jeune femme pose une question au maire sortant :

$\mathrm{JF}$ : «J'ai écrit plusieurs fois à la mairie pour un emploi dans une école. Personne ne répond. J'ai passé un diplôme et personne ne répond. ».

Le maire: «je ne vais pas vous donner un emploi comme ça. On va noter votre nom, vous revenez en septembre. Je vous mentirais si je vous disais que je peux vous donner un emploi. ».

Lors de deux autres réunions de quartier le même jour, il répète la même antienne "Je n'ai rien dans les poches. Mais je peux régler des problèmes collectifs ${ }^{37} \gg$. Il n' hésite cependant pas à accuser son adversaire de telle pratique clientéliste: "On m'a dit que lui, il va au marché, quelqu'un vient le voir pour lui demander du travail, il dit, viens à la mairie le 10 mars [lendemain des élections] à $10 \mathrm{H}$. Et il dit ça à plusieurs personnes. ${ }^{38}{ }{ }$

Cette dénégation peut être interprétée comme une anticipation des critiques adverses en même temps qu'un aveu de l'inaptitude du PSG à

\footnotetext{
${ }^{35}$ Observations, Cayenne, 6 mars 2008

${ }^{36}$ Idem.

${ }^{37}$ Idem.

${ }^{38}$ Idem.
} 
maintenir un niveau satisfaisant de redistribution de ressources. Quoi qu'il en soit, l'échange clientélaire ouvert est désormais disqualifié et bouleverse les habitudes de campagne des grands partis qui en ont usé.

De son côté, la liste conduite par Rodolphe Alexandre ne dénonce pas directement le «clientélisme » mais démontre sa capacité à défaire l'emprise du PSG et de la mairie sortante sur les agents municipaux qui seraient pris dans les filets du «clientélisme » municipal. Lors du débat télévisé sur télé Guyane, il affirme qu'une « circulaire faisant pression » sur les agents de la mairie leur aurait été envoyée avec leur bulletin de paye. Lors de plusieurs meetings, certains agents de la commune qui subiraient des pressions de la part de l'équipe en place et du PSG et qui se sont ralliés à la liste Alexandre sont présentés comme des héros et des trophées arrachés au PSG qui en aurait fait sa clientèle captive. Lors d'un grand meeting place des Palmistes $^{39}$, l'animateur introduit en ces mots un jeune homme, présenté comme « un simple citoyen», agent de la Mairie de Cayenne: "Il ose, parce qu'il est digne, orgueilleux, fier, comme vous les Cayennais ».

C'est le mouvement Walwari - lors d'entretiens avec ses membres, et dans des tracts - qui est le plus virulent à l'encontre des pratiques du PSG à la mairie de Cayenne ("clientélisme» et fraudes électorales). Dans un tract, il est affirmé que « Seule la liste 'réalisons l'harmonie' pourra rompre avec l'immoralité qui caractérise la gestion du maire actuel, et de son devancier, son premier adjoint »; la liste Alain Chaumet n'est pas en reste : « Je veux mettre un terme à la gestion néfaste et incohérente conduite par la municipalité sortante » (tract). Néanmoins, le personnel municipal reste une cible électorale privilégiée et un enjeu électoral car on connaît sa capacité à mobiliser (il est devenu un agent électoral du fait de la mobilisation du PSG depuis 40 ans) : le mouvement Walwari répète à plusieurs reprises vouloir « redonner leur dignité » aux agents municipaux (tract); de son côté, Rodolphe Alexandre accuse son principal adversaire de faire courir des bruits sur une éventuelle «chasse aux sorcières» au sein des agents municipaux s'il était élu (Télé Guyane, 4 mars 2008).

A Saint-Laurent du Maroni, on observe également une dénonciation du «clientélisme » dans ses formes les plus criantes. Les listes opposées à Léon Bertrand dénoncent le «clientélisme » et les promesses non tenues du

${ }^{39}$ Observations, Cayenne, $1^{\text {er }}$ mas 2008. 
maire sortant ; les Verts qui appartiennent à la liste EUC, ont fait de ce thème leur cheval de bataille depuis quatre ans, Brigitte Wyngaarde, candidate aux cantonales et ancienne candidate aux législatives, avait par exemple brandi un tee-shirt Léon Bertrand sur un plateau télévisé en lui demandant pourquoi il ne distribuait ces tee-shirts qu'aux Indiens et Noirsmarrons. Léon Bertrand ne cesse pas de distribuer des tee-shirts dans les villages amérindiens, mais il a intégré le caractère illégitime de certaines pratiques qui disparaissent de cette campagne :

«Il existait des pratiques, des manières de faire les campagnes et notamment avec les populations autochtones, bushinengué [noirs-marrons], amérindienne et petit à petit les choses ont tendance à se normaliser. [...] C'était vraiment culturel, c'était des dons, c'était pas des dons matériels. Ce n'est pas vrai, souvent on a parlé de dons matériels, non. Mais quand on allait dans une ethnie, il fallait amener de la bière, du rhum, du tabac, mais ça, c'est un rituel qui n'existait pas que pour la politique, on a tendance à faire changer tout ça.» ${ }^{40}$

Et surtout le maire de St Laurent substitue de nouveaux agents clientélaires à d'anciens intermédiaires jugés peu compétents ou dont la redistribution des biens clientélaires apparaît inefficace. Ainsi l'adjoint amérindien sortant, membre de la majorité du maire depuis 1983 n'est pas reconduit sur la liste ; sa belle-sœur, plus jeune et parlant mieux le français, l'y remplace. Elle explique :

«Le maire lui disait 'tu fais ça, ça, ça' et quand le maire lui demandait s'il avait fait, il disait 'oui'. Le maire lui faisait confiance. Mais moi, par derrière j'arrivais et je disais 'non', il ne l'a pas fait et du coup, il se faisait remonter les bretelles. Il n'a pas du tout apprécié. J'ai dit au maire 'Ecoutez, c'est mon beau-frère mais quand il faut faire le travail, il faut le faire et les gens se plaignent et viennent me voir. Je leur dis d'aller le voir, il leur dit, je vais écrire au maire mais il ne le fait pas'. C'est pour ça qu'il n'est pas sur la liste parce qu'il y a eu du laisseraller pour les logements sociaux. »

\footnotetext{
${ }^{40}$ Entretien directeur de campagne de Léon Bertrand, op.cit.
} 
De la même manière, sans rompre les liens avec certains capitaines (chefs coutumiers amérindiens et bushinengé), le maire recourt à d'autres intermédiaires dotés de plus de compétences scolaires et administratives. En effet certains capitaines ont tendance à accaparer pour eux et leurs familles les biens donnés par le maire, délaissant une partie des habitants des villages qui s'en plaignent à la mairie. Auparavant, les liens de parenté permettaient une distribution équitable des ressources allouées par l'administration qui profitaient à l'ensemble du groupe familial. Mais avec la croissance démographique, ce mode de redistribution est devenu obsolète. Un habitant rapporte ainsi que Léon Bertrand s'est fâché contre le capitaine de son village :
«Le maire a toujours donné, fait venir des choses mais le capitaine garde pour lui et ses enfants, le reste ne profitait pas. Le capitaine demandait toujours des camions de sable juste pour mettre autour de chez lui, c'était juste pour lui, pas pour des travaux dans le village. Le maire s'en est rendu compte, du comportement du capitaine. Il lui a dit mais pourquoi tu veux toujours du sable, tu n'as pas besoin d'autres choses pour le village? Il vient moins souvent, fait venir ses adjoints. A la réunion, j'ai pris la parole, le capitaine m'a coupé la parole [...] Le maire s'est fâché : 'laissez parler les jeunes' !»

Le maire désavoue l'accaparement personnel des biens de la mairie et l'absence de projet de développement du village. En effet, en raison de l'accroissement démographique et de l'urbanisation, la commune intervient de plus en plus dans les villages amérindiens où elle met en place des équipements et des services publics. Dès lors que la commune y mène des opérations, elle a besoin d'intermédiaires susceptibles de les accompagner, or certains capitaines n'ont pas les compétences administratives pour cela. De ce fait les relations entre la commune et les villages ne peuvent plus se limiter au seul couple maire/capitaine et s'ouvrent à de nouveaux intermédiaires plus compétents. L'échange clientélaire ne devient donc pas obsolète avec la modernisation administrative ${ }^{41}$, à Saint-Laurent du Maroni, il s'y adapte en renouvelant ses agents.

\footnotetext{
${ }^{41}$ Jean-Louis Briquet montre bien comment, en Corse, le clientélisme s'est perpétué dans un contexte d'intense transformation des activités politiques. Jean-Louis Briquet, La tradition en mouvement : clientélisme et politique en Corse, Paris, Belin, 1997.
} 
Enfin, la liste de Léon Bertrand attaque le «clientélisme » des listes adverses. Ainsi une colistière amérindienne de la liste UMP dénonce un colistier d'une liste de gauche qui aurait promis des boissons alcoolisées à un amérindien alcoolique. "Il lui a dit qu'il lui remplirait le frigo», «On n'accepte plus cela dans les villages $»^{42}$.

2) L'échange clientélaire, condition d'efficacité de la mobilisation électorale et ressort de la diversité

A Saint-Laurent du Maroni, malgré leur dénonciation, les pratiques clientélaires demeurent très prégnantes, en particulier auprès des Amérindiens et Noirs-marrons. Avant chaque réunion de quartier, dans l'ensemble du territoire, un ou deux agents électoraux, dont un certain nombre d'employés précaires de la commune, préparent la venue du maire pour lequel l'affluence aux réunions est importante. La maîtrise territoriale du maire demeure ainsi très forte et le maire moque d'ailleurs l'absence d'un tel réseau chez ses adversaires. La relation clientélaire est multiforme. Dans certains villages amérindiens et noirs-marrons, elle se fonde sur des liens de patronage ancien entre les capitaines de village et le maire. Dans de nombreux villages, lors des réunions, les capitaines demandent aux habitants de voter pour le maire et démontrent leurs proximité et loyauté au maire. Au village Pierre, le capitaine Pierre dit qu'il faut voter pour Léon Bertrand «parce qu'il est de chez nous » (la mère du maire était Amérindienne) et lui tient l'épaule pendant toute la réunion. Le capitaine d'un village noirsmarrons dit que tout le village votera pour «Léon », pour «papa Bertrand » et que «les esprits voteront également pour lui ». A chaque fois, la réunion a d'ailleurs été minutieusement préparée, table avec napperon et chaise en bois attendant Léon Bertrand. La relation clientélaire est ici d'autant plus efficace qu'elle est ancienne : le capitaine Pierre soutient le maire depuis 1983, le capitaine du village noirs-marrons a toujours travaillé avec le maire qui en échange le soutient pour qu'il obtienne le titre officiel de capitaine qu'il n'a pas encore. Dans d'autres cas, la relation de loyauté est moins ancienne. Ainsi à Balaté, la réunion publique est organisée par un jeune homme du village, agent précaire à la mairie. Or c'est précisément l'échange clientélaire pérenne entre le maire et les représentants des communautés

${ }^{42}$ Observation réunion publique au village Prospérité, 4 mars 2008. 
noirs-marrons et amérindiens qui assurent la loyauté de ces communautés au maire, qui y bénéficie d'un soutien sans égal avec ses adversaires. Lors des porte-à-porte, les électeurs de Saint-Laurent du Maroni sont parfois assez contestataires avec les candidats de l'opposition, rarement avec le maire et ses colistiers car le porte-à-porte suppose justement d'avoir des biens clientélaires à distribuer, apanage des sortants ${ }^{43}$. C'est ainsi aux challengers qui sont dépourvus de ressources clientélaires qu'on reproche de faire des promesses et de ne pas les tenir :

«Électeur: Cette dame [une candidate], si elle dit et qu'elle ne fait pas, alors c'est un mensonge qu'on ne peut pas pardonner parce que les enfants grandissent jour après jour.

Candidate EUC : c'est vrai que Léon Bertrand a promis plein de choses, la sécurité, des emplois, et qu'il ne l'a pas fait, c'est vrai qu'il a menti, il a menti sur plein de choses, mais si on ne donne pas l'opportunité à d'autres...

Électeur: Mais qui n'a pas menti pour obtenir une voix [...]. Tous les ans, vous êtes de bons orateurs pour obtenir une voix. ${ }^{44}$

$\mathrm{Au}$ maire, on fait peu de reproches et lorsqu'on le fait, on a la prudence de toujours montrer des gages de loyautét ${ }^{45}$. L'échange clientélaire est ainsi une ressource primordiale qui permet au maire d'attirer de nombreux agents électoraux issus des communautés et de démontrer son insertion dans l'ensemble des communautés de la ville alors que les listes

\footnotetext{
${ }^{43}$ Lefebvre Rémi. «Ethnographie des porte-à-porte de Martine Aubry », p. 203, in : Lagroye Jacques, Lehingue Patrick, Sawicki Frédéric (eds.), Mobilisations électorales: le cas des élections municipales de 2001. Paris : PUF, 2005.

${ }^{44}$ Échange entre un électeur du village amérindien de Paddock et les candidats de la liste EUC, Paddock, le 6 mars 2008.

${ }^{45}$ Une habitante au maire : «Avant je l'appelais Monsieur Léon, maintenant je ne l'appelle plus Monsieur Léon [elle parle de Léon Bertrand]. Je l'appelle Léon, parce que [...] je n'ai plus de respect pour lui, c'est pour cela que je l'appelle Léon maintenant. Parce qu'il a fait quelque chose de pas bien ici, la dernière fois qu'il est venu à Sparouine, on lui a demandé de mettre quelqu'un, un natif de Sparouine, sur sa liste, et il a dit oui, et il ne l'a pas fait. A Sparouine, nous ne parlons pas bien français, et celui qui serait natif de Sparouine serait l'intermédiaire entre Bertrand et Sparouine et cela il ne l'a pas fait. Il a menti, il n'a pas tenu sa promesse, il avait dit qu'il le ferait mais il ne l'a pas fait. Alors on accepte de voter pour lui mais il doit le faire cette fois-ci. On va voter pour lui, mais il ne doit pas mentir. » Réunion publique au village Sparouine, le 7 mars 2008.
} 
adverses ont du mal à pénétrer les villages et quartiers noirs-marrons. Plusieurs réunions des deux listes de gauche sont ainsi annulées dans ces quartiers, faute de public, d'autres listes n'en organisent pas, vraisemblablement par anticipation du manque de public et se contentent de passer avec les voitures sono.

\section{Conclusion}

Ainsi, ces deux villes, marquées par des histoires évidemment singulières, apparaissent comme des espaces politiques dans lesquels les acteurs politiques se réapproprient des registres de légitimation de l'espace central, pour surmonter des contraintes et mutations locales, remettant ainsi en cause certaines pratiques anciennes. Nous avons vu le rôle de deux entrepreneurs politiques, l'un challenger, l'autre établi, mais tous deux multi-positionnés et, pour l'un, implanté dans le champ central, dans ce processus d'importation et de traduction locale. Cette intégration relative des espaces municipaux, notamment en contexte ultra-marin, fonctionne comme un réservoir inépuisable d'inventivité politique. En effet, loin d'être une appropriation mécanique et un simple placage du discours métropolitain, la mobilisation du registre de la diversité s'articule avec d'autres pratiques de légitimation telle que la notabilité et/ou la relation de clientèle. Cette enquête nous informe donc autant sur l'émergence de ce nouveau registre et les transformations de la vie politique locale que sur la reproduction des formes de domination traditionnelles et la capacité de perpétuation des anciennes loyautés dans un contexte de changement social et politique intense. Mais là encore cette reproduction diffère selon les contextes locaux et les ressources des candidats puisqu'à Saint-Laurent du Maroni, la domination traditionnelle se nourrit des deux registres - diversité et compétence - alors qu'à Cayenne, ces derniers viennent disqualifier les loyautés clientélaires. 


\section{RESUME}

La transformation des registres de légitimation politique en Guyane : une approche comparative des campagnes municipales à Cayenne et Saint-Laurent du Maroni

Villes à très forte croissance démographique, Cayenne et Saint-Laurent du Maroni, sont des sites particulièrement riches pour l'observation des transformations des formes de légitimation des élus locaux. Singulièrement disputées du fait de la longévité des maires en place (ou de leur parti) et de certains signes avant-coureurs de l'effritement de leur popularité, les élections municipales de 2008 révèlent le recours, par les listes en concurrence, à des attributs de la modernité politique empruntés à l'espace politique central. La construction d'un nouveau rapport à la population insistant sur la représentation de la «diversité » et des «communautés » composant les populations de Cayenne et de Saint-Laurent du Maroni relève de cette démarche. Cet article montre combien ce registre de la diversité et de la communauté peut servir à exacerber les clivages dans un contexte de fortes oppositions entre les candidats, au delà des pratiques de campagne qui peuvent se ressembler. L'article montre ensuite que seule la permanence de pratiques anciennes, même revues à la marge, peut expliquer que ce registre symbolique soit aussi efficace. Ainsi, si compétence a rimé, lors de la campagne, avec communauté, c'est que la première permet une sélectivité sociale forte des élites politiques derrière un discours toujours aussi modernisateur. De plus, si les «communautés» (souvent en fait, les minorités), sont mises à l'honneur, ce sont avant tout les pratiques clientélaires, certes renouvelées, qui permettent de maintenir une loyauté sans faille et de garantir la longévité politique de certaines élites politiques. 


\begin{abstract}
The transformation of the registers of political legitimation in French Guiana :

a comparative approach of the municipal campaigns in cayenne and Saint- Laurent du Maroni
\end{abstract}

Cayenne and Saint-Laurent du Maroni as cities of very high increase in population are especially rich spots for the observation of how the forms of legitimation of the local councillors are changing. Extremely contested for the longevity of service of the mayors (or of their party) and for some forerunners announcing the crumbling of their popularity, the municipal elections in 2008 reveal the mobilization of attributes of modern politics, borrowed from the central political space, in using competition rolls. The construction of a new relationship with the population, insisting on the representation of "diversity" and "communities" composing the populations of Cayenne and Saint-Laurent du Maroni is a product of this approach. This article shows in what extent the registers of diversity and of community contribute to intensify the split in a context of strong opposition between the candidates, beyond the campaign practices that can be alike. The article points out next that only the permanency of ancient practices, even revised at the margin, can explain that this symbolic register is so efficient. Thus if, during the campaign, competency rhymed with community, the first term allows a strong social selectivity of the political elite behind a still as modernizing discourse. Moreover, if the "communities" (being in fact often the minorities) are much in evidence, it is especially due to clientelist practices, even if they are revived, that make possible the preservation of unfailing loyalty and the guarantee of political longevity of some political elite. 